\title{
Operando X-ray Investigation of Electrode/Electrolyte Interfaces in Model Solid Oxide Fuel Cells
}

\author{
Sergey Volkov, ${ }^{\dagger, \ddagger}$ Vedran Vonk, ${ }^{* \dagger}$ Navid Khorshidi, ${ }^{\#}$ Dirk Franz, ${ }^{\dagger}{ }^{\ddagger}$ Markus Kubicek, ${ }^{\S}$ Volkan Kilic, \\ Roberto Felici, ${ }^{\perp}$ Tobias M. Huber, ${ }^{\S}$ Edvinas Navickas, ${ }^{\S}$ Ghislain M. Rupp, ${ }^{\S}$ Jürgen Fleig, ${ }^{\S}$ \\ and Andreas Stierle ${ }^{\dagger, 末}$ \\ ${ }^{\dagger}$ Deutsches Elektronen-Synchrotron DESY, Notkestr. 85, 22607 Hamburg, Germany \\ ${ }^{\ddagger}$ Fachbereich Physik, Universität Hamburg, Jungiusstr. 9, 20355 Hamburg, Germany \\ \#Max-Planck-Institut für Intelligente Systeme (former Max-Planck-Institut für Metallforschung), Heisenbergstr. 3, 70569 Stuttgart, \\ Germany \\ ${ }^{\S}$ Vienna University of Technology, Institute of Chemical Technologies and Analytics, Getreidemarkt 9, A-1060 Vienna, Austria \\ "Universität Siegen, D-57072 Siegen, Germany \\ ${ }^{\perp}$ ESRF, F-38000 Grenoble, France
}

Supporting Information

ABSTRACT: We employed operando anomalous surface X-ray diffraction to investigate the buried interface between the cathode and the electrolyte of a model solid oxide fuel cell with atomic resolution. The cell was studied under different oxygen pressures at elevated temperatures and polarizations by external potential control. Making use of anomalous X-ray diffraction effects at the $\mathrm{Y}$ and $\mathrm{Zr} \mathrm{K}$ edges allowed us to resolve the interfacial structure and chemical composition of a (100)-oriented, $9.5 \mathrm{~mol} \%$ yttria-stabilized zirconia (YSZ) single crystal electrolyte below a $\mathrm{La}_{0.6} \mathrm{Sr}_{0.4} \mathrm{CoO}_{3-\delta}$ (LSC) electrode. We observe yttrium segregation toward the YSZ/LSC electrolyte/electrode interface under reducing conditions. Under

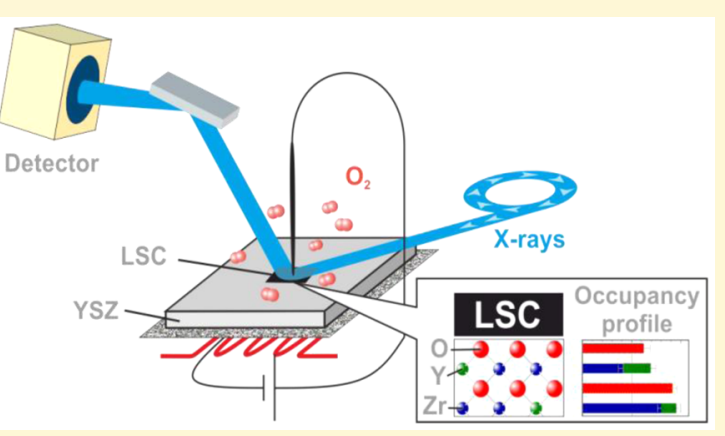
oxidizing conditions, the interface becomes $\mathrm{Y}$ depleted. The yttrium segregation is corroborated by an enhanced outward relaxation of the YSZ interfacial metal ion layer. At the same time, an increase in point defect concentration in the electrolyte at the interface was observed, as evidenced by reduced YSZ crystallographic site occupancies for the cations as well as the oxygen ions. Such changes in composition are expected to strongly influence the oxygen ion transport through this interface which plays an important role for the performance of solid oxide fuel cells. The structure of the interface is compared to the bare YSZ(100) surface structure near the microelectrode under identical conditions and to the structure of the YSZ(100) surface prepared under ultrahigh vacuum conditions.

\section{INTRODUCTION}

Solid oxide fuel cells (SOFCs) are very promising as a source for sustainable and renewable power generation. Despite the efforts of researchers worldwide, SOFC technology has not reached widespread commercial success yet. A more detailed, atomic scale understanding of the elementary, interface controlled processes of SOFCs is necessary to tailor their performance and lifetime. SOFCs' basic principle of operation is as follows: oxygen is reduced to $\mathrm{O}^{2-}$ on the cathode side, and then oxygen ions are transported through the electrolyte to the anode side where they react with hydrogen from pure hydrogen or hydrocarbons to form water and release electrons, which travel back to the cathode side through the external circuit giving electrical power.

Conventional SOFCs are operated at elevated temperatures $(1000-1250 \mathrm{~K})$ which results in higher costs of the cell and specific material requirements (durability, electrode/electrolyte interface stability). ${ }^{1}$ Bottlenecks for the SOFC operation are the cathodic oxygen exchange reaction on the surface of the electrode or the oxygen transport through the electrode/ electrolyte interface. ${ }^{2}$ Lowering operational temperatures while keeping a high ion transport rate is one of the key tasks in today's SOFCs development. ${ }^{2}$ Regarding the electrode material, perovskites are suitable candidates for intermediate temperature range $(800-1000 \mathrm{~K})$ SOFCs. ${ }^{1,3}$ The mixed ionic-electronic conductor $\mathrm{La}_{1-x} \mathrm{Sr}_{x} \mathrm{CoO}_{3-\delta}$ (LSC) is a promising cathode material, due to its high oxygen conductivity and fast oxygen surface kinetics. ${ }^{3,4}$ In order to independently investigate the cathode side of the fuel cell and to be able to deduce factors and parameters influencing SOFC performance, cathode model

Received: January 29, 2016

Revised: May 11, 2016

Published: May 11, 2016 
systems are often studied. Conventional model systems usually consist of a perovskite thin film as an electrode deposited on yttria-stabilized zirconia (YSZ) single crystals serving as electrolyte. A further microstructuring of the perovskite thin films has the advantage of a negligible effect of the counter electrode and thus no need for a reference electrode. ${ }^{5}$ SOFC model electrodes are traditionally studied by electrochemical impedance spectroscopy (EIS) and time-of-flight secondary ion mass spectrometry (TOF-SIMS). EIS delivers fingerprint type information on the underlying transport processes under operando conditions, whereas TOF-SIMS is a destructive technique employed ex situ after the experiment to establish laterally resolved oxygen diffusion profiles via isotope labeling. ${ }^{2}$ In addition, state-of-the-art ambient pressure photoemission spectroscopy can reveal the chemical binding state of surface atoms under fuel cell operation conditions. ${ }^{6}$

Despite recent progress made by the use of model microelectrodes, there is still a lack of knowledge on the atomic structure and chemical composition of buried electrode/ electrolyte interfaces of such systems. At these interfaces oxygen incorporation or release takes place under transport conditions, which may be accompanied by a change of the interfacial cation composition, detrimental for transport properties of the interface. It is a challenge to obtain an atomic-scale picture during the processes occurring at the interface, because a nondestructive probe with high penetration power as well as high interfacial sensitivity is needed, which is compatible with the harsh operation conditions of atmospheric oxygen pressure and elevated temperatures. Since the electrode is typically a few hundreds of nanometers thick, any charged probe such as electrons or ions disqualify for interface operando studies. Here we apply anomalous surface X-ray diffraction (SXRD) using a microfocused X-ray beam to reveal the interfacial electrode/electrolyte atomic structure and chemical composition as a function of temperature, ambient pressure, and polarization under operation conditions. These challenging experiments allowed us to resolve the complete 3D interfacial structure and composition with subatomic resolution despite the presence of background scattering and fluorescence from the $200 \mathrm{~nm}$ thick LSC electrode and the YSZ substrate. The structural information is contained in the so-called crystal truncation rods (CTRs), which are lines of diffracted intensity in reciprocal space arising from the abrupt termination of the polished YSZ single crystal substrate. Such atomic-scale crystallographic information on one particular interface in the SOFC can serve as very important input into further modeling of the oxygen transport mechanism. At the moment, most of the information available under operando conditions stems from impedance measurements, which are determined by all the interfaces in the SOFC. By including the details of one particular interface the transport models can be further refined leading to a better microscopic understanding.

Using synchrotron radiation the $\mathrm{X}$-ray energy can be precisely tuned to the $\mathrm{Y}$ and $\mathrm{Zr}$ absorption edges, thereby varying the scattering contrast between the two elements and retrieving the layer-wise composition, disclosing information on the segregation profiles. In addition the synchrotron X-ray beam can be effectively focused down to the size of the microelectrode, thereby enabling to study the electrode/ electrolyte interface and also the bare YSZ surface by lateral sample translation. Using highly intense X-rays from an undulator source makes a fast data acquisition possible, which is indispensible for such operando studies. Previous anomalous
SXRD experiments revealed that the bare and clean YSZ(111) surface in ultrahigh vacuum (UHV) exhibits an Y enrichment under reducing conditions and high temperature annealing. ${ }^{7-10}$

To mimic the transport processes in a real fuel cell, we polarized the cathode microelectrode with respect to a porous Pt counter electrode below the single crystal YSZ(100) electrolyte under oxygen atmosphere at elevated temperatures, allowing controlled inward and outward oxygen ion transport. The cathode consists of a polycrystalline complex oxide electrode in contact with a single crystal electrolyte, a situation which is representative for a real SOFC. Our results disclose an operation condition dependent variation in the interfacial structure, Y composition and cation vacancy concentration as compared to bulk YSZ. Such nonstochiometry is expected to have strong impact upon the interfacial oxygen ion conductivity. For comparison, a free part of the YSZ(100) surface was investigated, which exhibits compositions and relaxations that do not depend on the oxygen pressure and external voltage.

\section{EXPERIMENTAL DETAILS AND DATA ANALYSIS}

Two samples were subjects of our study: the first was a cathode model system consisting of a $\mathrm{La}_{0.6} \mathrm{Sr}_{0.4} \mathrm{Co}_{3-\delta}$ (LSC) microelectrode on an yttria-stabilized zirconia (YSZ) single crystal $\left(9.5 \mathrm{~mol} \%\right.$ of $\left.\mathrm{Y}_{2} \mathrm{O}_{3}\right)$ in (100) orientation and miscut of $<0.1^{\circ}$. The second was a pristine (100) YSZ single crystal with identical specifications from the same manufacturer. Measurements on the clean YSZ(100) substrate are compared with those taken from the bare part beside the microelectrode from the first sample. A $200 \mathrm{~nm}$ thin LSC film was deposited on the YSZ(100) substrate by pulsed laser deposition (PLD) at $900 \mathrm{~K}$ and 0.04 mbar $\mathrm{O}_{2}$ pressure followed by wet chemical etching with $0.1 \mathrm{~mol} / \mathrm{L} \mathrm{HCl}$ in deionized water to prepare a squared $400 \times 400 \mu \mathrm{m}^{2}$ microelectrode. The well-defined geometry of the electrode facilitated the interpretation of impedance spectroscopy measurements performed before and after the experiment demonstrating that electrode survived the X-ray experiment without significant beam damage.

The sample with LSC microelectrode on top was studied at the European Synchrotron Radiation Facility (ESRF), beamline ID03, ${ }^{11}$ by means of anomalous SXRD at the $\mathrm{Y}$ and $\mathrm{Zr}$ K-edges under controlled oxygen environment and temperature, as well as applied bias voltage, in a dedicated mobile vacuum chamber for combined solid state electrochemistry-XRD experiments. The experiment was performed in $z$ axis diffraction mode with fixed incidence angle, allowing to keep the beam footprint on the sample surface constant. ${ }^{12}$ By convention, a unit cell is chosen whereby the reciprocal (continuous) $L$ direction is parallel to the surface normal and therfore along the CTR directions. The in-plane lattice parameters span the surface and determine the (integer) diffraction indices $(h, k)$ of each CTR. Diffraction data are collected by obtaining integrated intensities at several $(h, k, l)$ positions in reciprocal space and extracting structure factors $F(h k l)$, which can be compared with calculated ones stemming from model structures. The chamber is equipped with a turbo molecular pump, which is supported by an external pre-pump to allow ultrahigh vacuum conditions, with a ceramic heater (sample temperature up to $\sim 1100 \mathrm{~K}$ ), leak valves for controlled gas dosing, and two pressure gauges for vacuum and elevated pressure ranges, a piezo translation stage to position a contact tip, an optical microscope to locate the electrodes, and an X-ray transparent beryllium window. Platinum paste was brushed on the back side of the YSZ single crystal and served as counter-electrode for the electrical measurements. The sample was then clamped onto an Inconel sample holder. The principle scheme of the experimental setup is depicted in Figure 1. Anomalous SXRD data sets were collected by measuring crystal truncation rods (CTRs) at the K-edge energies of $\mathrm{Y}$ and $\mathrm{Zr}$, respectively (17.038 and $17.998 \mathrm{keV})$, using a $2 \mathrm{D}$ detector in stationary mode. ${ }^{13}$ The graphite analyzer in front of the detector 


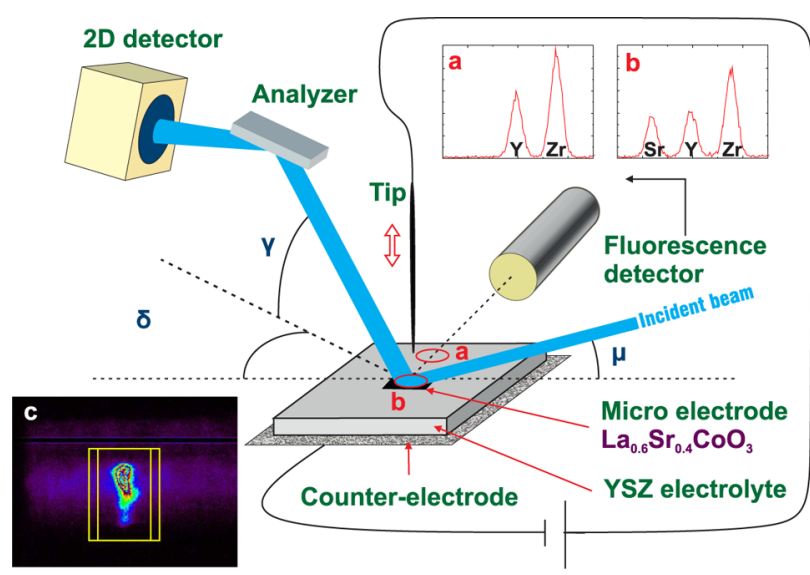

Figure 1. Scheme of the experimental setup. The YSZ crystal with LSC microelectrode on top and the brushed Pt as a counter-electrode at the bottom are placed on a heating stage. The tip is attached to a piezo-electric translation stage, which is positioned on the upper part of the chamber. Tip and counter-electrode can be connected to the impedance analyzer and a power source via feedthroughs of the vacuum chamber. A Ge fluorescence detector was placed at $90^{\circ}$ to the $\mathrm{X}$-ray beam in the horizontal plane in order to minimize elastic scattering. An analyzer (graphite (0001) crystal) was put in signal path to suppress the fluorescence background. (a) Fluorescence spectrum from a sample area without the electrode; (b) fluorescence spectrum from a sample area with the electrode; (c) an image of CTR signal together with a region of interest (ROI)-yellow box.

allowed an efficient fluorescence background suppression. When the beam energy is tuned to one of the absorption edges, the real part of the atomic form factor of the corresponding atoms is reduced. (In addition, the imaginary part of the complex atomic scattering factor increases due to absorption processes.) Exploiting this anomalous diffraction effect allows us to distinguish between $\mathrm{Y}$ and $\mathrm{Zr}$, which have almost identical atomic form factors at $\mathrm{X}$-ray energies away from their absorption edges. By including data measured at several X-ray energies in the structure refinement, it is possible to determine the individual compositions with a higher accuracy. ${ }^{10}$

In order to illuminate only the area underneath one electrode, the X-ray beam was strongly focused by a Kirkpatrick-Baez (KB) optics resulting in a beam size of $5.5 \mu \mathrm{m}$ (vertical) and $5.7 \mu \mathrm{m}$ (horizontal). Such a small beam cross section ensures that its footprint at the incident angle of $0.9^{\circ}$ was slightly smaller than the electrode size (400 $\times 400 \mu \mathrm{m}^{2}$ ). The beam stayed on the same spot of the electrode throughout the measurements involving sample rotation which was verified by tracking the $\mathrm{Sr}$ fluorescence signal from the LSC electrode with an energy-dispersive $\mathrm{Ge}$ detector placed perpendicular to the direction of the primary beam; see Figure 1 . The electrode was first characterized under reducing conditions, and then we used a step-bystep approach to reach operando conditions, while recording crystal truncation rod data at each step to follow the buried interface evolution. An extensive set of CTRs was taken at five different conditions (altogether, 1216 structure factors for both energies): reducing condition $\left(p=1.0 \times 10^{-7} \mathrm{mbar}, 300\right.$ and $\left.775 \mathrm{~K}\right)$, oxidizing conditions $\left(p\left(\mathrm{O}_{2}\right)=18 \mathrm{mbar}, 775 \mathrm{~K}\right)$ and operational conditions $\left(p\left(\mathrm{O}_{2}\right)=18 \mathrm{mbar}, 775 \mathrm{~K},-500 \mathrm{mV}\right.$ and $\left.+250 \mathrm{mV}\right)$. The measurements under reducing conditions and cathodic bias were thus close to the thermodynamic stability limit of LSC, i.e., (surface) phase decomposition. ${ }^{14}$ During cathodic and anodic polarization the electrical current through the electrode was monitored confirming that the model fuel cell was operating. The bare single crystal YSZ(100) reference sample was studied at the Max Planck beamline at the Ångström-Quelle Karlsruhe (ANKA) ${ }^{15}$ by SXRD at $10 \mathrm{keV}$ photon energy under UHV conditions after annealing at $700 \mathrm{~K}$ in oxygen atmosphere $\left(p\left(\mathrm{O}_{2}\right)=1.0 \times 10^{-5} \mathrm{mbar}\right)$ for $120 \mathrm{~min}$. All the data presented here were analyzed using the software package ROD. ${ }^{16,17}$ The program allows refinement of surface relaxations, roughnesses, and Debye-Waller factors. A special version of ROD was used, featuring the possibility to refine anomalous CTR data sets taken at different energies at the same time.

\section{RESULTS AND DISCUSSION}

An overview of the SXRD experiments from the LSC/ YSZ(100) interface is given in Figure 2, which represents the CTR data (open circles) measured at three different conditions $\left(p=1.0 \times 10^{-7}\right.$ mbar, $775 \mathrm{~K} ; p\left(\mathrm{O}_{2}\right)=18 \mathrm{mbar}, 775 \mathrm{~K} ; p\left(\mathrm{O}_{2}\right)=$ $18 \mathrm{mbar},-500 \mathrm{mV}$ ) and the fits (solid lines). (Additional data obtained for other conditions and for the free YSZ(100) surface in between the microelectrodes is shown in the Supporting Information. Also shown are the results obtained for the clean YSZ(100) surface in UHV.) Significant changes in the CTR signals for the different conditions are discernible, related to structural changes at the interface as uncovered by the fit to the data and discussed in the following. We used the idealized structure model of $\mathrm{CaF}_{2}$ type, $F m \overline{3} m$ space group, where $\mathrm{Y}$ and $\mathrm{Zr}$ are placed statistically on bulk positions according to the nominal formula $\mathrm{Y}_{0.174} \mathrm{Zr}_{0.826} \mathrm{O}_{0.193}$ and a cubic lattice constant



Figure 2. CTR data from the electrode/electrolyte interface and fits for three different conditions: $300 \mathrm{~K}, p=1.0 \times 10^{-7} \mathrm{mbar}(\mathrm{blue} \mathrm{line}) ; 775 \mathrm{~K}$, $p\left(\mathrm{O}_{2}\right)=18 \mathrm{mbar}$ (green line), $775 \mathrm{~K}, p\left(\mathrm{O}_{2}\right)=18 \mathrm{mbar}, U=-500 \mathrm{mV}$ (red line). One data set consists of eight CTRs-four taken at Zr K-edge (a), and the other four taken at Y K-edge (b). All eight CTRs taken under each particular condition are fitted simultaneously. On the $(1,-1)$ rod the signal was cut by one of the supporting steel rods of the Be window. 

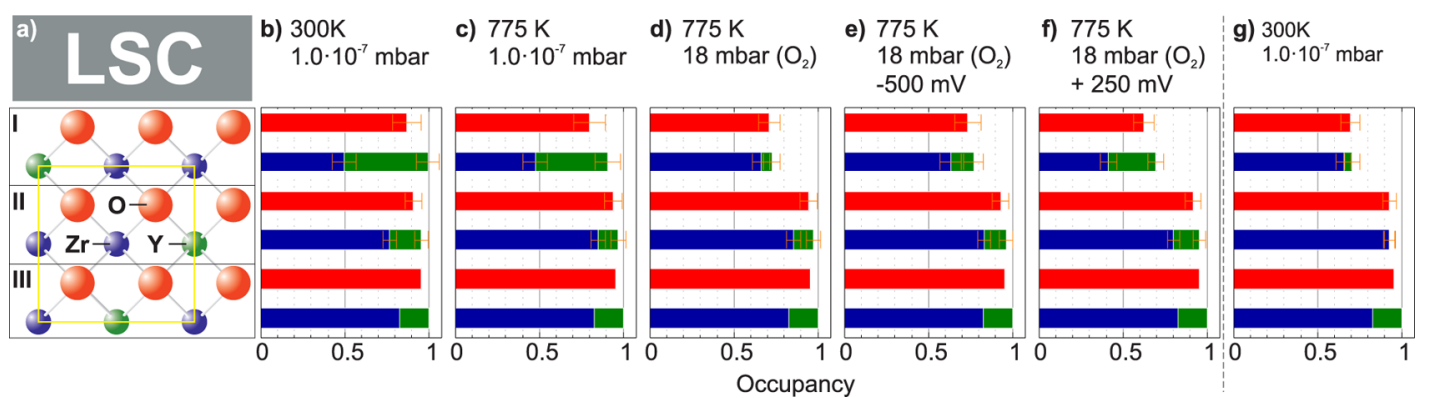

Figure 3. (a) Side view of the first three atomic layers (I, II, III) and the LSC film placed on the oxygen (red spheres) terminated YSZ surface. Yttrium atoms (green spheres) are randomly placed on zirconium (blue spheres) sites within the lattice. (b-f): Chemically resolved occupancy profiles of the first three atomic bilayers of YSZ(100) below the LSC electrode for the conditions indicated, where the third layer represents bulk values as a reference and the first and second layer occupancies were fitted. The YSZ bulk unit cell is marked with yellow. The color code of bar plots corresponds to the one for the atoms. (g) Reference profile for a bare YSZ(100) surface area far away from the electrode.

of $a=5.145 \AA$ deduced from the experiment. Note that the LSC films do not contribute to the CTR signal because they exhibit a polycrystalline structure. ${ }^{18}$ YSZ, with its complex defect structure, ${ }^{19}$ can also be described by an elaborate socalled $\mathrm{Zr}$-shift model, ${ }^{20}$ but, as discussed previously in ref 8 , a simple zirconia fluorite model is sufficient to describe $\mathrm{X}$-ray data taken at momentum transfers covered in our experiment.

As a starting model for the electrode-covered as well as the free YSZ(100) surface an oxygen termination was chosen. The YSZ crystal structure is polar along its $\langle 100\rangle$ direction due to the alternating stacking of oxygen and metal ion layers ${ }^{21}$ (see Figure 3a). At the LSC/YSZ interface charge compensation to overcome the diverging dipole moment can take place via bonding to atoms from the LSC layer. During our operando studies the free part of the sample surface is likely to be partially hydroxylated, which can also stabilize the surface. When in vacuum, such intrinsically unstable polar surfaces are known to commonly show reconstructions, in order to decrease their otherwise very high surface energy. Based on the results from a low energy ion scattering study ${ }^{22}$ the surface oxygen atoms are reported to occupy nonbulk positions, situated at metal bridge sites above metal ions from the second metal layer below the surface. Density functional theory calculations ${ }^{21}$ support the experimentally obtained $(1 \times 1)$ surface reconstruction but only together with $\mathrm{Y}$ segregation. However, our UHV data taken after annealing up to $750 \mathrm{~K}$ do not allow us to make a clear distinction between a bulk oxygen-terminated or the proposed $(1 \times 1)$ model. $^{23}$ In any case all our fit results do indicate a surface morphology composed of nanoscaled islands of uniform well-defined $0.25 \mathrm{~nm}$ height, almost identical to what was found for the YSZ(111) surface. ${ }^{9,24}$

Atomic site occupancies $(\theta)$, displacements $(\Delta)$, and Debye-Waller factors $(B)$ are those three structural parameters refined within our model. Three parameters $(\theta, \Delta, B)$ were varied in the first atomic layer, only two $(\theta, \mathrm{B})$ are used for the second atomic layer, from the third layer on all the parameters are fixed to the bulk YSZ values of the ideal fluorite model taken from ref 14 (see Figure 3 and Table 1). The refinement also included physically acceptable limits for each parameter used in the fit. Since the scattering contribution of oxygen is significantly weaker as compared to zirconium and yttrium, the oxygen occupancy parameters were fixed with respect to those of $\mathrm{Y}$ and $\mathrm{Zr}$ atoms according to chemical formulas $\mathrm{Y}_{2} \mathrm{O}_{3}$ and $\mathrm{ZrO}_{2}$. By doing this we restrain the oxygen occupancy parameter and assume electroneutrality. To reduce the number of parameters which are highly correlated, the same Debye-
Waller parameter and displacement parameter was used for $\mathrm{Y}$ and $\mathrm{Zr}$ atoms within one atomic layer. Debye-Waller factors with lower limits and starting values for all atom types were equal to the corresponding bulk values. ${ }^{20}$ Refining three atomic layers and more did not show any considerable fit improvement.

As an important fit result, the atomic-layer-resolved chemical profiles at the LSC/YSZ interface were obtained, as summarized in Figure 3. First, under oxygen poor conditions $\left(p=10^{-7} \mathrm{mbar}, 300 \mathrm{~K}\right)$ we find that the first atomic layer has a composition $\mathrm{Y}_{0.5} \mathrm{Zr}_{0.5}$, while the second layer is already close to the bulk chemical composition, indicating a strong $\mathrm{Y}$ segregation in the as prepared sample. To the best of our knowledge this is the first observation of interfacial $Y$ segregation at a buried interface. The strong $\mathrm{Y}$ segregation to the top of YSZ layer presumably took place during PLD deposition of the LSC layer at $900 \mathrm{~K}$ and 0.04 mbar $\mathrm{O}_{2}$ pressure. $\mathrm{Y}$ segregation under reducing conditions is in accordance with measurements and $a b$ inito thermodynamics calculations on free YSZ surfaces, ${ }^{9,25-27}$ while the exact level of $\mathrm{Y}$ is most likely also affected by the LSC layer on top. In comparison the free YSZ surface several millimeters away from the electrode shows a topmost layer consisting of uniform islands, which is seen in the total coverage of about $70 \%$. Furthermore, these islands as well as the second layer beneath them are depleted in $\mathrm{Y}$, and the trend is that under more oxidizing conditions $\mathrm{Y}$ starts to segregate to the near-surface region (see Supporting Information). Although this trend is observed, the total Y concentration is very close to the error bar and presumably the near-surface composition hardly changes. The island structure and coverage agree very well with the results from the UHV prepared YSZ(100) surface (see Supporting Information). The presence of islands, which can be interpreted as clustering of atomic vacancies, may contribute to the polarity compensation of the YSZ(100) surface.

The high Y occupancy in the top YSZ layer at the electrode/ electrolyte interface remains present at $775 \mathrm{~K}$ under reducing conditions $\left(p=1.0 \times 10^{-7}\right.$ mbar $)$, while around $10 \%$ cation vacancies (likely together with oxygen vacancies) are formed. When switching to oxidizing conditions $\left(p\left(\mathrm{O}_{2}\right)=18 \mathrm{mbar}\right)$ at $775 \mathrm{~K}$ the cation occupancy strongly changes. Much less $\mathrm{Y}$ is observed in the first atomic layer, while an increase in $\mathrm{Zr}$ concentration is present and the cation vacancy concentration becomes more substantial. This clearly indicates that cations in YSZ are mobile even at such comparatively low temperatures. A similar analysis carried out on the electrode material would be 
desirable and in principle possible if single crystal epitaxial LSC films were used. Nevertheless, some consequences of the results presented here can be discussed. Measurements upon applied voltage between the tip and the sample to mimic the oxygen reduction reaction (ORR) $(-500 \mathrm{mV})$ and the reversed process $(+250 \mathrm{mV})$ again indicate that the YSZ/LSC interface is highly dynamic (Figure 3e,f). At $-500 \mathrm{mV}$ voltage the $\mathrm{Y} / \mathrm{Zr}$ composition at the interface is similar to the conditions from before without voltage applied, whereas at $+250 \mathrm{mV}$ the interface becomes enriched in $\mathrm{Y}$. This might be a consequence of the oxygen chemical potential changes in LSC upon positive voltage: Owing to the slow kinetics of the electrochemcial surface reaction (here release of oxygen) the main chemical potential drop is at the surface, particularly upon cathodic voltage but also for moderate positive bias. ${ }^{28}$ Hence, positive bias voltage leads to an increase of the chemical potential in LSC and thus significantly lowers the oxygen vacancy concentration in LSC. Provided that oxygen transport across the LCS/YSZ interface is still close to equilibrium, this concentration change in LSC has to be counterbalanced by an electrostatic potential step (Nernst's equation) with positive charges in LSC and negative ones in YSZ. A possible mechanism for the substrate could be played by $\mathrm{Y}$ ions on $\mathrm{Zr}$ sites. In such a case an electroneutral calculation of the oxygen vacancy concentration in YSZ is no longer possible. We argue this is due to the fact that oxygen diffusion is faster in LSC than in YSZ, resulting in net reduction of the interface, which leads to a $\mathrm{Y}$ enrichment. This might be related to a reduced oxygen concentration at the LSC/YSZ interface under positive potential, due to the fact that the amount of oxygen vacancies in YSZ is directly related to the yttria concentration. Discussing the obtained cation concentrations in a more crude way as interfacial stoichiometry changes lead to the following considerations. It is known that optimum doping level for oxygen conduction in YSZ is close to that found in the substrate. $^{29}$ This means that as soon as the interfacial region deviates from this composition, it can be expected that the interface forms an additional diffusion barrier. According to our observation, the YSZ interfacial cation stoichiometry is almost identical to that of the bulk for negative bias. Indeed, impedance spectroscopy of the related ( $\mathrm{La}, \mathrm{Sr})(\mathrm{Co}, \mathrm{Fe}) \mathrm{O}$ system has shown that there is a clear trend of the electrode/ electrolyte interface resistance and capacitance increasing when going from negative to positive bias voltage. ${ }^{28}$ The results presented here suggest that part of this trend can be explained by the near-surface stoichiometry changes and show that the method presented here can be used to give very detailed microscopic information on the origin of certain properties found in SOFCs. One important conclusion concerning the long-term stabililty of SOFCs at the maximum investigate temperature of $775 \mathrm{~K}$ is that the LSC/YSZ(100) smooth interface morphology is found to stay unaltered under oxygen transport conditions. It has been reported that, at higher temperatures above $970 \mathrm{~K}$, new phases like $\mathrm{SrZrO}_{3}$ can form which we did not observe in our experiments. ${ }^{30,31}$

Further evidence for the discussed Y segregation behavior comes from a close investigation of the interfacial relaxations. The first and second YSZ substrate metal ion layer directly at the electrolyte/electrode interface exhibits outward relaxations which may also partially be explained by the chemical bonding of the outermost oxygen layer to atoms from the LSC electrode (see Table 1). In contrast, the first metal ion layer at the free surface far away from the electrode shows slight inward 
relaxations for all conditions investigated, followed by the second layer with small outward relaxations, in agreement with the UHV prepared YSZ(100) surface (see also Supporting Information).

To obtain more insight into the correlation of structural and chemical changes at the interface, it is instructive to compare the $\mathrm{Y} / \mathrm{Zr}$ ratio and the interfacial relaxations as a function of the experimental conditions, as plotted in Figure 4. Interestingly,

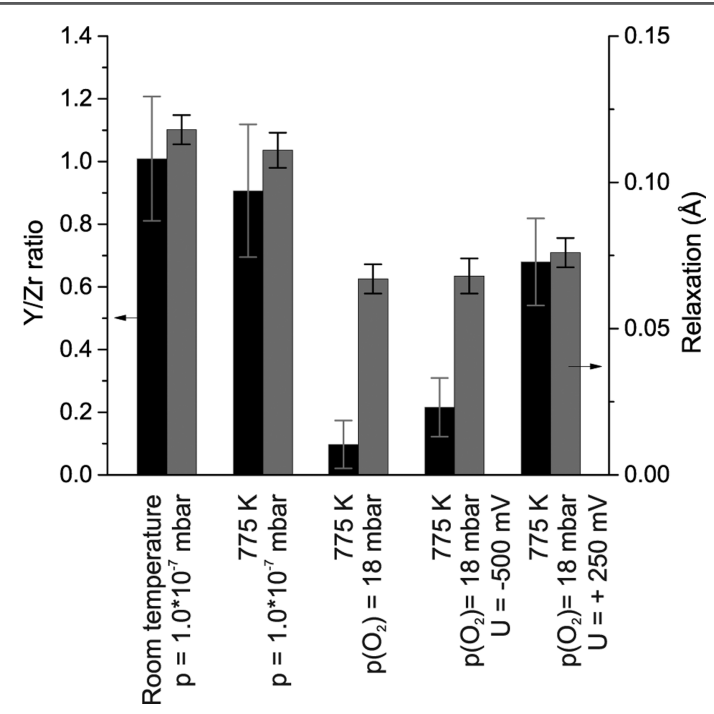

Figure 4. $\mathrm{Y} / \mathrm{Zr}$ ratio (left $y$ axis) and interfacial cation out-of-plane relaxations (right $y$ axis) for different experimental conditions.

there is a clear trend visible: for higher $\mathrm{Y} / \mathrm{Zr}$ ratios, the outward relaxations are more pronounced. This is inline with the increased ionic radius of $\mathrm{Y}^{3+}$ of $1.02 \AA$ as compared to $0.84 \AA$ for $\mathrm{Zr}^{4+}$, giving additional evidence for the observed $\mathrm{Y}$ segregation. ${ }^{32}$ Finally, the isotropic Debye-Waller parameters, which were refined for "below the electrode" data only, exhibit enhanced values compared to the bulk likely because of some local distortions at the interface. Here no systematic trend is discernible, which may be related to the high error bars for the Debye-Waller parameters.

\section{CONCLUSIONS}

In our study we demonstrated that anomalous SXRD can resolve the chemical composition of deeply buried interfaces of SOFC model systems under operational conditions with atomic resolution. The presented investigation of a polycrystalline LSC model microelectrode on a YSZ(100) substrate showed that the $\mathrm{Y}$ cation occupancy at the electrode/electrolyte interface strongly depends on the sample environment and the applied potential. The interface was found to be Y rich after LSC film deposition; subsequent exposure to oxygen in the mbar pressure regime at $775 \mathrm{~K}$ strongly reduced the $\mathrm{Y}$ concentration at the interface, which is traced back to the complex defect chemistry and thermodynamics of LSC in contact with YSZ. Upon bias voltage application further changes are observed, indicating enhanced $\mathrm{Y}$ concentration at the interface for positive bias which is expected to be detrimental for the oxygen ion transport through the interface. The $\mathrm{Y}$ segregation behavior is supported by outward cation interfacial relaxation which correlates with the amount of $\mathrm{Y}$ at the interface. The LSC/YSZ(100) interface is found to stay atomically smooth under oxygen transport conditions. Reference measurements from a bare YSZ(100) surface area indicate stable surface composition and relaxations. The observed interfacial defects and variation from bulk stochiometry for different conditions calls for further theoretical investigations to disentangle their role for the rate limitation of the oxygen reduction reaction. Future experiments on epitaxial electrode layers will allow the additional investigation of the gas phase/electrode interface under oxygen incorporation or removal conditions.

\section{ASSOCIATED CONTENT}

\section{S Supporting Information}

The Supporting Information is available free of charge on the ACS Publications website at DOI: 10.1021/acs.chemmater.6b00351.

Additional details concerning the results, such as structure parameters, fits, and residuals of refinement (PDF)

\section{AUTHOR INFORMATION}

\section{Corresponding Author}

*(V.V.) E-mail: vedran.vonk@desy.de.

\section{Notes}

The authors declare no competing financial interest.

\section{ACKNOWLEDGMENTS}

The authors gratefully acknowledge financial support from the German Science Foundation (DFG) under Project No. STI 554/1-1 and the Austrian Science Fund (FWF) under Project No. F45 SFB Functional Oxide Surfaces and Interfaces (FOXSI). We thank Olga Perkova for designing a new dedicated mobile chamber for combined solid state electrochemistry-XRD experiments.

\section{REFERENCES}

(1) Brandon, N.; Skinner, S.; Steele, B. Recent Advances in Materials for Fuel Cells. Annu. Rev. Mater. Res. 2003, 33, 183-213.

(2) Fleig, J. Solid Oxide Fuel Cell Cathodes: Polarization Mechanisms and Modeling of the Electrochemical Performance. Annu. Rev. Mater. Res. 2003, 33, 361-382.

(3) Adler, S. B. Factors governing Oxygen Reduction in Solid Oxide Fuel Cell Cathodes. Chem. Rev. 2004, 104, 4791-4843.

(4) Mobius, H. H. On the History of Solid Electrolyte Fuel Cells. J. Solid State Electrochem. 1997, 1, 2-16.

(5) Fleig, J. Microelectrodes in Solid State Ionics. Solid State Ionics 2003, 161, 279-289.

(6) Zhang, C.; Grass, M. E.; McDaniel, A. H.; DeCaluwe, S. C.; El Gabaly, F.; Liu, Z.; McCarty, K. F.; Farrow, R. L.; Linne, M. A.; Hussain, Z.; Jackson, G. S.; Bluhm, H.; Eichhorn, B. W. Measuring fundamental properties in operating solid oxide electrochemical cells by using in situ X-ray photoelectron spectroscopy. Nat. Mater. 2010, 9, 944-949.

(7) Vonk, V. Surface Structure Refinement Including Anomalous Crystal Truncation Rods. J. Appl. Crystallogr. 2011, 44, 1217-1221.

(8) Perret, E.; Park, C.; Fong, D. D.; Chang, K.-C.; Ingram, B. J.; Eastman, J. A.; Baldo, P. M.; Fuoss, P. H. Resonant X-ray Scattering Studies of Epitaxial Complex Oxide Thin Films. J. Appl. Crystallogr. 2013, 46, 76-87.

(9) Vonk, V.; Khorshidi, N.; Stierle, A.; Dosch, H. Atomic Structure and Composition of the Yttria-stabilized Zirconia (111) Surface. Surf. Sci. 2013, 612, 69-76.

(10) Vonk, V.; Huijben, J.; Kukuruznyak, D.; Stierle, A.; Hilgenkamp, H.; Brinkman, A.; Harkema, S. Polar-discontinuity-retaining $A$-site Intermixing and Vacancies at $\mathrm{SrTiO}_{3} / \mathrm{LaAlO}_{3}$ Interfaces. Phys. Rev. B: Condens. Matter Mater. Phys. 2012, 85, 045401. 
(11) Balmes, O.; van Rijn, R.; Wermeille, D.; Resta, A.; Petit, L.; Isern, H.; Dufrane, T.; Felici, R. The ID03 Surface Diffraction Beamline for In-situ and Real-time X-ray Investigations of Catalytic Reactions at Surfaces. Catal. Today 2009, 145, 220-226.

(12) Feidenhans'l, R. Surface Structure Determination by X-ray Diffraction. Surf. Sci. Rep. 1989, 10, 105-188.

(13) Schleputz, C.; Herger, R.; Willmott, P.; Patterson, B.; Bunk, O.; Bronnimann, C.; Henrich, B.; Hulsen, G.; Eikenberry, E. Improved data acquisition in grazing-incidence $\mathrm{X}$-ray scattering experiments using a pixel detector. Acta Crystallogr., Sect. A: Found. Crystallogr. 2005, 61, 418-425.

(14) Kuhn, M.; Hashimoto, S.; Sato, K.; Yashiro, K.; Mizusaki, J. Oxygen nonstoichiometry and thermo-chemical stability of $\mathrm{La}_{0.6} \mathrm{Sr}_{0.4} \mathrm{Co}_{3-\delta}$. J. Solid State Chem. 2013, 197, 38-45.

(15) Stierle, A.; Steinhauser, A.; Ruhm, A.; Renner, F.; Weigel, R.; Kasper, N.; Dosch, H. Dedicated Max-Planck Beamline for the in situ Investigation of Interfaces and Thin Films. Rev. Sci. Instrum. 2004, 75, 5302-5307.

(16) Vlieg, E. ROD: a Program for Surface X-ray Crystallography. J. Appl. Crystallogr. 2000, 33, 401-405.

(17) Vlieg, E. Integrated Intensities Using a Six-Circle Surface X-ray Diffractometer. J. Appl. Crystallogr. 1997, 30, 532-543.

(18) Kubicek, M.; Huber, T. M.; Welzl, A.; Penn, A.; Rupp, G. M.; Bernardi, J.; Stöger-Pollach, M.; Hutter, H.; Fleig, J. Electrochemical Properties of $\mathrm{La}_{0.6} \mathrm{Sr}_{0.4} \mathrm{CoO}_{3-\delta}$ Thin Films Investigated by Complementary Impedance Spectroscopy and Isotope Exchange Depth Profiling. Solid State Ionics 2014, 256, 38-44.

(19) Hull, S. Superionics: Crystal Structures and Conduction Processes. Rep. Prog. Phys. 2004, 67, 1233.

(20) Ishizawa, N.; Matsushima, Y.; Hayashi, M.; Ueki, M. Synchrotron Radiation Study of Yttria-stabilized Zirconia, $\mathrm{Zr}_{0.758} \mathrm{Y}_{0.242} \mathrm{O}_{1.879}$. Acta Crystallogr., Sect. B: Struct. Sci. 1999, 55, 726-735.

(21) Ballabio, G.; Bernasconi, M.; Pietrucci, F.; Serra, S. Ab-initio Study of Yttria-stabilized Cubic Zirconia Surfaces. Phys. Rev. B: Condens. Matter Mater. Phys. 2004, 70, 075417.

(22) Nishimura, T.; Toi, H.; Hoshino, Y.; Toyoda, E.; Kido, Y. Surface Structure of $\mathrm{Y}_{2} \mathrm{O}_{3} 9.5 \mathrm{~mol} \%$-stabilizedZrO $\mathrm{Zr}_{2}(001)$ Determined by High-Resolution Medium-Energy Ion Scattering. Phys. Rev. B: Condens. Matter Mater. Phys. 2001, 64, 073404.

(23) Khorshidi, N. In-situ X-ray Studies of Model Electrode Surfaces for Solid Oxide Fuel Cells. Ph.D. thesis, University of Stuttgart, 2010.

(24) Morrow, S.; Luttrell, T.; Carter, A.; Batzill, M. High Temperature Scanning Tunneling Microscopy of Purely Ion Conducting Yttria-stabilized Zirconia (YSZ). Surf. Sci. 2009, 603, L78.

(25) Theunissen, G.; Winnubst, A.; Burggraaf, A. Surface and Grainboundary Analysis of Doped Zirconia Ceramics Studied by AES and XPS. J. Mater. Sci. 1992, 27, 5057-5066.

(26) Lahiri, J.; Mayernick, A.; Morrow, S. L.; Koel, B. E.; van Duin, A. C. T.; Janik, M. J.; Batzill, M. Modification of Active Sites on YSZ(111) by Yttria Segregation. J. Phys. Chem. C 2010, 114, 59905996.

(27) Wang, X.-G. Yttrium Segregation and Surface Phases of Yttriastabilized Zirconia (111) Surface. Surf. Sci. 2008, 602, L5-L9.

(28) Baumann, F. S.; Fleig, J.; Habermaier, H.-U.; Maier, M. Impedance spectroscopic study on well-defined $(\mathrm{La}, \mathrm{Sr})(\mathrm{Co}, \mathrm{Fe}) \mathrm{O}_{3-\delta}$ model electrodes. Solid State Ionics 2006, 177, 1071-1081.

(29) Nakamura, A.; Wagner, J. Defect Structure, Ionic-Conductivity, and Diffusion in Yttria Stabilized Zirconia and related Oxide Electrolytes with Fluorite Structure. J. Electrochem. Soc. 1986, 133, $1542-1548$.

(30) Yamamoto, O.; Takeda, Y.; Kanno, R.; Noda, M. Perovskitetype Oxides as Oxygen Electrodes for High-Temperature Oxide FuelCells. Solid State Ionics 1987, 22, 241.

(31) Sase, M.; Ueno, D.; Yashiro, K.; Kaimai, A.; Kawada, T.; Mizusaki, J. Interfacial Reaction and Electrochemical Properties of Dense (La,Sr) $\mathrm{CoO}_{3-\delta}$ Cathode on YSZ(100). J. Phys. Chem. Solids 2005, 66, 343.
(32) Shannon, R. D. Revised Effective Ionic Radii and Systematic Studies of Interatomic Distances in Halides and Chalcogenides. Acta Crystallogr., Sect. A: Cryst. Phys., Diffr., Theor. Gen. Crystallogr. 1976, 32, 751-767. 